\title{
FISIOTERAPIA RESPIRATÓRIA NO PÓS-OPERATÓRIO DE CIRURGIA ABDOMINAL ALTA: UMA REVISÃO DE LITERATURA
}

\author{
RESPIRATORY PHYSIOTHERAPY IN THE POSTOPERATIVE PERIOD OF HIGH ABDOMINAL \\ SURGERY: A REVIEW OF LITERATURE
}

\author{
Daísa Carla Bezerra Silva ${ }^{a}$, Luciano Santos da Silva Filho ${ }^{b}$ \\ adaisacarla@hotmail.com, bluciano0306@hotmail.com \\ Universidade Potiguar - Mossoró (RN), Brasil
}

Data de recebimento do artigo: 25/09/2017

Data de aceite do artigo: 25/10/2017

\section{RESUMO}

Introduçáo: A fisioterapia respiratória é uma alternativa terapêutica que atua tanto na prevenção quanto no tratamento de complicaçóes pulmonares pós-operatórias e objetiva um restabelecimento precoce da função pulmonar. Objetivos: Debater sobre as informaçôes mais recentes, em literaturas específicas, acerca da fisioterapia respiratória no pós-operatório de cirurgia abdominal alta, bem como confrontar e expor opinióes de diversos autores e estudos. Materiais e métodos: Caracteriza-se como uma pesquisa bibliográfica do tipo descritiva, baseada em coleta de dados feita entre janeiro de 2017 e março de 2017. O levantamento bibliográfico foi composto a partir do acesso às bases de dados SciElo, PubMed, LILACS, Bireme, BVS e FIEP Bulletin. O critério de inclusão foi a seleçấo de estudos que abordavam a temática em questão (como ensaios clínicos) até o ano de 2017. Já os critérios de exclusão foram: artigos publicados antes de 2005; com foco em treinamento pré-operatório; com direcionamento exclusivo para a avaliação de volumes pulmonares; que tratavam apenas das CPP decorrentes das cirurgias abdominais alta; e que fossem estudos de revisão. Resultados: Dos estudos encontrados, seis abordaram a espirometria de incentivo, quatro trataram da cinesioterapia respiratória convencional, três apresentaram a ventilação mecânica (um pelo método invasivo e os outros dois pela máscara de pressão expiratória positiva) e sete examinaram a fisioterapia respiratória de maneira generalizada. Conclusão: Com este estudo foi possível discutir as principais técnicas de fisioterapia respiratória utilizadas no pós-operatório de cirurgia abdominal alta, entretanto, náo foi possível eleger uma terapia que fosse essencial para o tratamento. Por outro lado, todos os estudos ressaltam a importância da fisioterapia para redução ou prevençáo de complicaçóes pulmonares.

Palavras-chaves: Reabilitação; cuidados pós-operatórios; exercícios respiratórios.

\section{ABSTRACT}

Introduction: Respiratory physiotherapy is a therapeutic alternative that acts both in the prevention and treatment of postoperative pulmonary complications and aims at an early reestablishment of pulmonary function. Objectives: To discuss the most recent information, in specific literature, about respiratory physiotherapy in the postoperative period of high abdominal surgery, as well as to confront and present opinions of several authors and studies. Materials and methods: It is characterized as a bibliographic research of the descriptive type, based on a collection of data carried out between January 2017 and March 2017. The bibliographic survey was made based on the access to the database of SciElo, PubMed, LILACS, Bireme, BVS and FIEP Bulletin. Inclusion criteria were to select studies that approached the subject in question (such as clinical trials) until the year 2017. In its turn, exclusion criteria were: articles published before 2005; articles with an exclusive focus on preoperative training; articles exclusively for the evaluation of pulmonary volumes; articles that dealt only with the pulmonary complications resulting from high abdominal surgeries; and articles classified as review studies. Results: Six of the studies found 
incentive spirometry, four treated conventional respiratory kinesiotherapy, three presented mechanical ventilation (one by the invasive method and the other two by the positive expiratory pressure mask), and seven analyzed respiratory physiotherapy in a generalized manner. Conclusion: With this study, it was possible to discuss the main techniques of respiratory physiotherapy used in the postoperative period of high abdominal surgery, but it was not possible to choose a therapy as the most essential for the treatment. On the other hand, all studies emphasize the importance of physiotherapy for the reduction or prevention of pulmonary complications.

Keywords: Rehabilitation; postoperative care; respiratory exercises.

\section{Introdução}

O número de procedimentos cirúrgicos aumentou exponencialmente nos últimos anos. Acredita-se que 14 milhóes de cirurgias sejam realizadas no Brasil e que aproximadamente $40 \%$ das internaçóes hospitalares no país estejam relacionadas a algum tratamento cirúrgi$\mathrm{Co}^{1}$. Em países desenvolvidos, são realizados anualmente algo em torno de 500 a 1000 procedimentos cirúrgicos abdominais a cada 100.000 habitantes $^{2}$. Dentre as cirurgias, destacam-se as torácicas e abdominais alta, que consistem em métodos rotineiros para o tratamento de diversas doenças e para o diagnóstico de determinadas condiçôes ${ }^{3}$.

As cirurgias abdominais altas compreendem os procedimentos realizados nos quadrantes superiores da regiáo abdominal e possuem alto índice de complicaçóes pulmonares $(\mathrm{CPP})$ devido à proximidade do local de intervenção com o músculo diafragma e o tórax. A incidência de comprometimento pulmonar de relevância clínica varia de $5 \%$ a $30 \%$ no pós-operatório (PO) de cirurgias abdominais ${ }^{1,4}$, e a taxa de prevalência das complicações respiratórias varia de 17 a $88 \%$ nas cirurgias abdominais altas ${ }^{5-7}$. Os principais processos cirúrgicos na porção superior do abdômen são: a gastrectomia (utilizada para remoção de câncer gástrico, apresentando alta complexidade e elevada taxa de mortalidade) ${ }^{8,9}$; laparotomia (como secção abdominal para acesso a órgáos intra-abdominais ou como método para diagnóstico) ${ }^{9,10}$; colecistectomia (cirurgia para tratamento de alteraçóes na vesícula biliar e que dentre as cirurgias abdominais é a mais frequente, apresentando baixos índices de mortalidade e de complicaçóes $)^{1,9}$; colecistectomia laparoscópica (variação da colecistectomia, sendo minimamente invasiva, apresentando tempo reduzido de cirurgia e permanência hospitalar, bem como gastos hospitalares menores) $)^{1,11,12}$; esofagectomia (procedimento em que é realizada uma ressecção de determinada porção do esôfago e que apresenta a maior taxa de CPP dentre as cirurgias abdominais) ${ }^{1,9,13}$; e gastroplastia (utilizada para tratamento da obesidade mórbida) ${ }^{9,14}$.

Assim, fatores como idade avançada ${ }^{15,16}$, sexo ${ }^{15}$, administração de opioides no intra-operatório ${ }^{15}$, tabagismo ${ }^{15,16}$, duração da anestesia ${ }^{15}$, doença cardiopulmonar preexistente $^{15,16}$, obesidade ${ }^{16,17}$, desnutriçãa ${ }^{13,16}$, tempo cirúrgico e técnica utilizada ${ }^{16,18}$ estão intimamente relacionados com a ocorrência de complicaçóes pulmonares no pós-operatório de cirurgias abdominais altas $(\mathrm{CAA})^{13,15-18 .}$

Dentre as principais complicações, destaca-se o desenvolvimento de atelectasia, hipoxemia e pneumonia, afetando cerca de $80 \%$ dos pacientes submetidos ao procedimento cirúrgico ${ }^{6,19,20}$. São encontrados casos de infecção traqueobrônquica, insuficiência respiratória aguda, tempo maior de ventilação mecânica e broncoespasmo ${ }^{19}$.

De acordo com Forgiarini et al. ${ }^{4}$, devido à inibição reflexa do nervo frênico pela manipulação visceral durante o procedimento, além da dor após a operação e o colapso alveolar, ocorre uma reduçáo de $50 \%$ a $60 \%$ da capacidade vital (CV) e de $30 \%$ da capacidade residual funcional (CRF) graças à disfunção diafragmática. A diminuição da CRF pode ser a causa da hipoxemia pelo aumento do fluxo sanguíneo, impedindo que ocorra a troca gasosa ${ }^{21}$. Também são observadas alterações nos volumes pulmonares, como na capacidade pulmonar total (CPT) e em volume corrente (VC), nos valores arteriais de pressão arterial de oxigênio $\left(\mathrm{PaO}_{2}\right)$ e na saturação de oxigênio $\left(\mathrm{SatO}_{2}\right)^{22}$.

Quanto à fisioterapia respiratória, trata-se de uma alternativa terapêutica que atua tanto na prevenção quanto no tratamento de complicaçóes pulmonares pós-operatórias ${ }^{4,20,23}$ e objetiva um restabelecimento precoce da função pulmonar ${ }^{24}$. Assim, técnicas fisioterapêuticas são utilizadas com o intuito de minimizar os riscos de complicaçóes pulmonares, por colaborarem para o aumento das capacidades pulmonar e residual funcional, resultando em índices cada vez menores de atelectasias ${ }^{19}$.

Desta forma, este estudo procura debater sobre as informaçóes mais recentes, em literaturas específicas, acerca da fisioterapia respiratória no pós-operatório de cirurgia abdominal alta, bem como confrontar e expor opiniōes de diversos autores e estudos. Tal objetivo surgiu da necessidade de entender como a fisioterapia respiratória pode proporcionar uma melhor e eficaz recuperação dos pacientes submetidos a esse tipo de procedimento, o qual afeta diretamente a integridade respiratória do indivíduo, já que a alta incidência de 
complicaçôes respiratórias nas cirurgias de abdômen superior possui correlação com internação hospitalar prolongada, índices de morbimortalidade, uso de medicação e altos custos hospitalares ${ }^{1,2,4}$.

\section{Metodologia}

O estudo em questáo caracteriza-se como uma pesquisa bibliográfica do tipo descritiva, que verifica estudos divulgados anteriormente, traça um quadro teórico e faz a estruturação conceitual para sustentar o desenvolvimento de pesquisa sobre a fisioterapia respiratória no pós-operatório de cirurgia abdominal alta. O trabalho foi realizado baseando-se na coleta de dados entre janeiro de 2017 e março de 2017.

O levantamento bibliográfico foi composto a partir do acesso às bases de dados SciElo, PubMed, LILACS, Bireme, BVS e FIEP Bulletin. As palavras-chave utilizadas nesse levantamento foram: reabilitação, cuidados pós-operatórios e exercícios respiratórios. O critério de inclusão foi a seleção de estudos que abordavam a temática em questão (como ensaios clínicos) até o ano de 2017. Já os critérios de exclusão foram: artigos publicados antes de 2005; com foco em treinamento pré-operatório; com direcionamento exclusivo para a avaliação de volumes pulmonares; que tratavam apenas das CPP decorrentes das cirurgias abdominais alta; e que fossem estudos de revisão.

\section{Resultados}

Foram selecionados vinte artigos que se enquadraram nos critérios estabelecidos pela pesquisa e, consequentemente, incluídos na revisão conforme apresentados em resumo no Quadro 1. Quanto ao tipo de tratamento fisioterapêutico utilizado, seis artigos abordaram a espirometria de incentivo ${ }^{5,17,24,25-27}$, quatro trataram da cinesioterapia respiratória convencional ${ }^{19,22,28,29}$, três estudos apresentaram a ventilação mecânica (um pelo o método invasivo ${ }^{18}$ e os outros dois pela máscara de pressão expiratória positiva ${ }^{24,30}$ ), e sete empregaram o atendimento em fisioterapia respiratória de maneira generalizada ${ }^{4,10,13,29,31}$. Quanto ao tipo de cirurgia abdominal alta pesquisada, foram encontrados: um artigo de laparotomia ${ }^{10}$, um artigo de esofagectomia ${ }^{13}$, dois artigos sobre gastroplastia ${ }^{17,24}$ e um artigo de colecistectomia laparoscópica ${ }^{25}$.

Quadro 1: Resumo de artigos encontrados

\begin{tabular}{|c|c|c|c|c|}
\hline TÍTULO & AUTOR & ANO & OBJETIVO & CONCLUSÃO \\
\hline $\begin{array}{l}\text { Inspirometria de incentivo e } \\
\text { breath stacking: repercussóes } \\
\text { sobre a capacidade inspirató- } \\
\text { ria em indivíduos submeti- } \\
\text { dos à cirurgia abdominal }\end{array}$ & Dias $^{5}$ & 2008 & $\begin{array}{l}\text { Comparar o volume inspiratório mo- } \\
\text { bilizado durante a técnica de breath } \\
\text { stacking com o volume na inspirome- } \\
\text { tria de incentivo em pacientes subme- } \\
\text { tidos à cirurgia abdominal }\end{array}$ & $\begin{array}{l}\text { A técnica de breath stacking mostrou-se } \\
\text { eficaz e superior à inspirometria de incenti- } \\
\text { vo para a geraçáo e sustentação de volumes } \\
\text { inspiratórios. }\end{array}$ \\
\hline $\begin{array}{l}\text { Fisioterapia respiratória no } \\
\text { pós-operatório imediato } \\
\text { em pacientes submetidos } \\
\text { à cirurgia abdominal alta: } \\
\text { ensaio clínico aleatório }\end{array}$ & Manzano $^{22}$ & 2008 & $\begin{array}{l}\text { Avaliar a eficácia da fisioterapia res- } \\
\text { piratória precoce no pós-operatório } \\
\text { imediato em pacientes submetidos à } \\
\text { cirurgia abdominal alta. }\end{array}$ & $\begin{array}{l}\text { A fisioterapia respiratória é efetiva na me- } \\
\text { lhora da saturação de oxi-hemoglobina em } \\
\text { pacientes observados na sala de recuperação } \\
\text { em cirurgia abdominal alta e não provoca } \\
\text { dor durante os exercícios. }\end{array}$ \\
\hline $\begin{array}{l}\text { Benefícios da cinesioterapia } \\
\text { respiratória no pós-ope- } \\
\text { ratório de colecistectomia } \\
\text { laparoscópica }\end{array}$ & Gastaldi $^{25}$ & 2008 & $\begin{array}{l}\text { Avaliar os efeitos da cinesioterapia } \\
\text { respiratória sobre a funçáo pulmonar } \\
\text { e a força muscular respiratória. }\end{array}$ & $\begin{array}{l}\text { A cinesioterapia respiratória contribuiu para } \\
\text { a recuperaçáo precoce da funçáo pulmonar e } \\
\text { da força muscular dos pacientes. }\end{array}$ \\
\hline $\begin{array}{l}\text { Efeito da cinesioterapia } \\
\text { respiratória em pacientes } \\
\text { submetidos à cirurgia } \\
\text { abdominal alta }\end{array}$ & Ribeiro $^{26}$ & 2008 & $\begin{array}{l}\text { Avaliar os efeitos da fisioterapia } \\
\text { respiratória sobre a função pulmonar } \\
\text { dos pacientes em PO de cirurgia } \\
\text { abdominal alta. }\end{array}$ & $\begin{array}{l}\text { A evolução do Grupo A foi semelhante ao } \\
\text { Grupo B (este, porém, com maior risco de } \\
\text { complicaçóes) sugerindo que a inclusão da } \\
\text { cinesioterapia foi benéfica. }\end{array}$ \\
\hline $\begin{array}{l}\text { Atendimento fisiotera- } \\
\text { pêutico no pós-operatório } \\
\text { imediato de pacientes } \\
\text { submetidos à cirurgia } \\
\text { abdominal }\end{array}$ & $\begin{array}{l}\text { Forgiarini } \\
\text { Júnior }\end{array}$ & 2009 & $\begin{array}{l}\text { Avaliar o efeito do atendimento } \\
\text { fisioterapêutico no pós-operatório } \\
\text { imediato de pacientes submetidos à } \\
\text { cirurgia abdominal. }\end{array}$ & $\begin{array}{l}\text { A fisioterapia realizada no PO imediato } \\
\text { reduziu a perda da funçáo pulmonar, a perda } \\
\text { da força muscular ventilatória e o tempo de } \\
\text { internação na sala de recuperação. }\end{array}$ \\
\hline
\end{tabular}


Quadro 1: Continuação.

\begin{tabular}{|c|c|c|c|}
\hline TÍTULO & AUTOR & ANO & OBJETIVO \\
\hline $\begin{array}{l}\text { Comparação entre inspi- } \\
\text { rometria de incentivo e } \\
\text { pressáo positiva expiratória } \\
\text { na funçâao pulmonar após } \\
\text { cirurgia bariátrica }\end{array}$ & $\begin{array}{l}\text { Barbalho- } \\
\text { Moulim }^{24}\end{array}$ & 2009 & $\begin{array}{l}\text { Comparar o efeito do EPAP e da } \\
\text { inspirometria de incentivo a fluxo } \\
\text { sobre a funçáo pulmonar após o } \\
\text { bypass gástrico em Y de Roux por } \\
\text { videolaparoscopia. }\end{array}$ \\
\hline $\begin{array}{l}\text { Inspirometria de incentivo } \\
\text { orientada a fluxo e padrôes } \\
\text { ventilatórios em pacien- } \\
\text { tes submetidos à cirurgia } \\
\text { abdominal alta }\end{array}$ & Chinali $^{27}$ & 2009 & $\begin{array}{l}\text { Verificar o comportamento da PImá } \\
\text { VEF1 e CVF antes e após o uso do } \\
\text { Respiron }{ }^{\circ} \text {; comparar o efeito da inspi } \\
\text { rometria de incentivo com os padróe } \\
\text { ventilatórios expansivos; constatar } \\
\text { presença de dor no pré-operatório e } \\
\text { no primeiro e no quarto dia de PO. }\end{array}$ \\
\hline $\begin{array}{l}\text { Tratamento fisioterapêu- } \\
\text { tico no pós-operatório de } \\
\text { laparotomia }\end{array}$ & Silva ${ }^{10}$ & 2010 & $\begin{array}{l}\text { Verificar a resposta do tratamento da } \\
\text { fisioterapia respiratória em pacientes } \\
\text { no primeiro dia pós-operatório de } \\
\text { laparotomia. }\end{array}$ \\
\hline $\begin{array}{l}\text { Fisioterapia respiratória } \\
\text { pós-operatória previne } \\
\text { complicaçôes respiratórias } \\
\text { em pacientes submetidos à } \\
\text { esofagectomia }\end{array}$ & Lunardi $^{13}$ & 2010 & $\begin{array}{l}\text { Comparar a frequência de complica- } \\
\text { çốes respiratórias em dois grupos de } \\
\text { pacientes submetidos à esofagecto- } \\
\text { mia, tendo um recebido fisioterapia } \\
\text { respiratória e o outro não. }\end{array}$ \\
\hline
\end{tabular}

Efeitos de exercícios respiratórios sobre o padrão respiratório e movimento toracoabdominal após gastroplastia

Efeitos de duas técnicas de incentivo respiratório na mobilidade toracoabdominal após cirurgia abdominal alta Ventilação com baixos volumes correntes durante cirurgia abdominal superior não melhora a função pulmonar pós-operatória

Exercícios respiratórios em cirurgia abdominal alta: revisão sistemática e metanálise

Grams $^{19}$

Tomich $^{17}$ 2010

Avaliar o padrão respiratório e o

\section{CONCLUSÃO}

A inspirometria de incentivo a fluxo exerceu melhores efeitos na manutenção do VC e na mobilidade diafragmática e toracoabdominal, enquanto a EPAP foi mais eficaz no restabelecimento do VRE.

A inspirometria de incentivo e os padrôes ventilatórios expansivos podem ter contribuído para a melhora dos valores espirométricos e PImáx, porém não houve diferenças significativas entre os métodos.

A fisioterapia respiratória contribuiu para a melhora do quadro respiratório, no que diz respeito ao VC e VM, e nas PImáx e PEmáx, porém foi evidenciado aumento do quadro de dor após a fisioterapia.

A fisioterapia respiratória após esofagectomia reduz as complicaçóes respiratórias e a necessidade de cuidados clínicos, mas não reduz o tempo de hospitalização.

Entre os exercícios respiratórios avaliados, a espirometria de incentivo orientado a volume forneceu os melhores resultados. exercícios respiratórios.

O grupo exercitado usando o dispositivo

Comparar duas técnicas de incentivo respiratório na recuperação da dinâmica toracoabdominal em pacientes submetidos à cirurgia abdominal alta. Voldyne ${ }^{\varpi}$ apresentou melhores resultados do que o grupo que treinou o padrão inspiratório em três tempos.

Comparar a função pulmonar em Treschan $^{18} 2012$ pacientes no PO submetidos a cirurgia abdominal superior, com VM em volumes correntes altos ou baixos.

A prolongada redução da função pulmonar após uma cirurgia abdominal não é amenizada pela baixa ventilação do volume corrente.

Realizar uma revisão sistemática de ensaios controlados aleatorizados e ensaios controlados quasi-aleatoriza-

Exercícios respiratórios provavelmente apresentam efeitos benéficos na força muscular respiratória em pacientes submetidos à CAA, no entanto a falta de estudos de boa qualidade comprometeu uma conclusão mais categórica.
Consenso sobre a gestão
fisioterápica de pacientes após cirurgia abdominal superior: uma abordagem Hanekom $^{29}$
Desenvolver um algoritmo de pragmática para interpretar evidência ambígua
A implementação de uma diretriz para a fisioterapia no período pós-operatório da ci- rurgia abdominal alta reduz a incidência de atelectasia e o tempo de internamento
conduta para a gestão pós-operatória de pacientes submetidos a cirurgias abdominais.
Avaliar a eficácia da implementação de uma diretriz de fisioterapia para Possa $^{30} 2013$ doentes submetidos à cirurgia abdo- minal superior (UAS) na redução da incidência de atelectasia e no tempo de internamento no PO. cios respiratórios na recuperaçáo da função pulmonar e prevenção da CPP após CAA.
Através de um processo de consenso um algoritmo de manejo clínico foi formulado. Este algoritmo pode agora ser usado por médicos para orientar a prática clínica nessa população.

A implementação de uma diretriz de fisioterapia para doentes submetidos a UAS resultou na redução da incidência de atelectasia e na reduçáo do tempo de internamento no PO. 
Quadro 1: Continuação.

\begin{tabular}{|c|c|c|c|c|}
\hline TÍTULO & AUTOR & ANO & OBJETIVO & CONCLUSÁO \\
\hline $\begin{array}{l}\text { Fisioterapia no pré e } \\
\text { pós-operatório de cirurgia } \\
\text { abdominal e torácica }\end{array}$ & Zancan $^{31}$ & 2013 & $\begin{array}{l}\text { Identificar e agrupar informaçóes sobre } \\
\text { o papel desempenhado pela fisioterapia } \\
\text { no tratamento de indivíduos que passa- } \\
\text { ram por cirurgia abdominal e torácica. }\end{array}$ & $\begin{array}{l}\text { Há necessidade de estudos bem desenhados } \\
\text { e com métodos e técnicas comparáveis, in- } \\
\text { cluindo-se aplicaçóes combinadas, conduzi- } \\
\text { das com o intuito de estabelecer programas } \\
\text { efetivos para diferentes perfis de pacientes. }\end{array}$ \\
\hline
\end{tabular}

(Prevenção de Infecção

Pulmonar pós-cirurgia abdominal alta com fisioterapia no pré-operatório): protocolo de estudo para um ensaio multicêntrico controlado randomizado

\section{LIPPSMAck POP}

Estimar o efeito que a educação e o treinamento de fisioterapia pré-operatória têm sobre a incidência de CPP após grandes CAA, quando em comparação a informativos.

Avaliar o efeito da fisioterapia pré-operatória no hospital e na Boden $^{2} 2015$ UTI, como nos custos hospitalares, na incidência de pneumonia, em admissóes não planejadas na UTI, no tempo para mobilização precoce, na prontidão para alta hospitalar e, seis semanas após a cirurgia, em complicaçóes relatadas pelo paciente por meio do Questionário de Qualidade de vida e Capacidade Funcional.

O uso de um dispositivo de pressão expiratória positiva oscilatória modificada reduz a febre e a duração da internaçáo em pacientes após cirurgia torácica e abdominal alta: um estudo randomizado

Comparação e avaliação dos efeitos da administração de ventilaçáo não-invasiva pós-operatória (CPAP Yağlığlu ${ }^{33}$

e BiPAP) na mecânica respiratória e troca gasosa em pacientes de CAA Comparação do inspirômetro de incentivo a fluxo e a volume na função pulmonar e na tolerância ao exercício em Cirurgia Abdominal Aberta: estudo clínico randomizado Zhang $^{32}$

Identificar os efeitos do uso regular de um dispositivo de PEP oscilatório na febre, contagem de glóbulos bran-

2015 cos, tempo de internação, mortalidade, custos de tratamento e necessidade de antibióticos em pacientes pós cirurgia torácica e abdominal alta.

Investigar os efeitos de dois diferentes métodos de CPAP e BiPAP e o suporte de oxigênio em ventilaçáo espontânea na mecânica respiratória, na troca gasosa e na lesão da máscara bucal e facial durante o pós-operatório de pacientes submetidos à CAA.

Avaliar os efeitos da inspirometria de incentivo a fluxo e a volume na Kumar $^{6}$ 2016 função pulmonar e na tolerância ao exercício de pacientes submetidos à cirurgia abdominal aberta.
Em adultos submetidos à cirurgia torácica e abdominal alta, o uso pós-operatório de um dispositivo PEP oscilatório resultou em menos casos de febre e menor tempo de internação. No entanto, a terapia com antibióticos e as despesas hospitalares totais não foram significativamente reduzidas por esta intervenção.

Administração profilática de suporte ventilatório pode prevenir a deterioração das funçôes pulmonares e hipóxia de pacientes com DPOC submetidos à CAA. Além disso, CPAP com fluxos baixos tem melhores efeitos na $\mathrm{PaO} 2, \mathrm{SpO} 2$ quando comparado a outras técnicas.

A espirometria de incentivo de fluxo e volume pode ser recomendada com segurança a pacientes submetidos à cirurgia abdominal aberta, uma vez que não foram registrados eventos adversos. Além disso, conduziu a uma melhora significativa na função pulmonar e à tolerância ao exercício.

Fonte: Elaboraçấo dos autores, 2017.

\section{Discussão}

Estudos revelam que as complicaçôes pulmonares no pós-operatório ocorrem numa frequência igual ou maior que as de ordem cardíacas. Entretanto, na maioria dos casos, o risco cardíaco é profundamente investigado quando comparado ao risco pulmonar ${ }^{34,35}$. A alta incidência de complicações respiratórias nas cirurgias de abdômen superior possui correlação com internação hospitalar prolongada, índices de morbimortalidade, uso de medicação e altos custos hospitalares ${ }^{1,2,4,6}$.

Gastaldi et al. ${ }^{25}$ desenvolveram seu estudo com dois grupos de indivíduos, um com 17 sujeitos submetidos à cinesioterapia respiratória (exercícios de respiração diafragmática, inspiração máxima sustentada e inspiração fracionada), e outro com 19 sujeitos (grupo controle) que não receberam tratamento. A partir das variáveis observadas, identificaram que os exercícios respiratórios 
proporcionaram uma recuperaçáo mais rápida dos volumes pulmonares e da força muscular respiratória desses pacientes. Em contrapartida, Boden et al. ${ }^{2}$ desenvolveram um procedimento operacional padrão (POP) de educação e treinamento fisioterapêutico pré-operatório aplicável a pacientes pós-CAA. O treino consistia em exercícios de respiração profunda e tosse durante $30 \mathrm{mi}-$ nutos. O ensaio é o primeiro que visa testar a hipótese de que a educação de fisioterapia pré-operatória previne as CPP desse perfil de pacientes, por isso seus resultados ainda são inconclusivos.

Manzano et al. ${ }^{22}$ realizaram um ensaio clínico aleatório com dois grupos: grupo controle e grupo fisioterapia respiratória, para identificar o atendimento fisioterapêutico ainda na sala de recuperação pós-anestésica de uma CAA. O controle foi submetido apenas à avaliação, e ao grupo de fisioterapia respiratória, além da avaliação, foi aplicada uma conduta fisioterapêutica baseada em exercícios como: passivo-localizado, respiração diafragmática profunda e de expansáo torácica, durante 30 minutos no primeiro e no segundo dia de PO. Ao categorizar os dados, identificaram que os resultados da espirometria pós-operatórios obtidos não apresentaram diferenças significativas. Afirmam ainda que a espirometria é controversa quando utilizada para mensurar a funçáo pulmonar. Ribeiro et al. ${ }^{26}$ também chegaram a essa conclusão em seu estudo com dois grupos, um realizando caminhada e tosse assistida, e outro realizando, além da caminhada e tosse assistida, cinesioterapia (exercícios respiratórios diafragmáticos, em tempos e inspiração sustentada). Sua pesquisa ressalta que os resultados da terapia foram satisfatórios, mas que não demonstraram diferença significativa entre os grupos.

Em pesquisa realizada por Trevisan et al. ${ }^{28}$ os pacientes foram distribuídos aleatoriamente em dois grupos, um submetido à espirometria de incentivo a volume (G1) e o outro ao padrão inspiratório em três tempos (G2). Constataram que o grupo submetido ao uso do Voldyne (G1) obteve resultados significativamente melhores da dinâmica toracoabdominal do que o G2, por ter melhora da expansibilidade e circunferência toracoabdominal mais significativas. Isso corroborou com o estudo de Tomich et al. ${ }^{17}$, no qual exercício diafragmático e espirometrias de incentivo orientadas a fluxo (EIOF) e a volume (EIOV) foram realizados em ordem aleatória em pacientes após gastroplastia, a fim de saber o efeito desses exercícios sobre o padrão respiratório e movimento toracoabdominal. Eles observaram que a EIOV forneceu os melhores resultados, uma vez que permitiu inspiraçóes mais lentas e profundas.

Kumar et al. ${ }^{6}$, em seu estudo clínico randomizado com 50 pacientes, dividiram igualmente dois grupos, um que recebeu tratamento com inspirômetro a fluxo e o outro a volume. Todos os pacientes foram avaliados no pré-operatório e nos cinco dias de pós-operatório. Identificaram que houve um decréscimo da função pulmonar no primeiro dia pós-cirúrgico e melhora significativa nos dias subsequentes em ambos os grupos, numa comparação ao pré-operatório. $\mathrm{O}$ ensaio apresentou também que os dois grupos aumentaram significativamente a distância percorrida no teste de caminhada de 6 minutos no pós-operatório, numa comparação com as distâncias no pré-cirúrgico.

Barbalho-Moulim et al. ${ }^{24}$, ao realizar um estudo comparativo entre a espirometria de incentivo a fluxo e o efeito da pressão positiva expiratória na função pulmonar após cirurgia bariátrica, observaram que no grupo inspirômetro (GI) houve redução com significância estatística do volume de reserva inspiratório (VRI) e expiratório (VRE), já no GE (grupo EPAP - expiratory positive dirway pressure ou pressão positiva expiratória na via aérea) encontraram valores menores com significância estatística das variáveis VRI e VC, entretanto, o VRE teve uma discreta redução. Em relação à mobilidade diafragmática, no GI houve redução apenas na hemicúpula esquerda e no GE ocorreu redução em ambas hemicúpulas e na mobilidade toracoabdominal, sem diferenças estatísticas nos níveis avaliados.

Dias et al. ${ }^{5}$, em seu ensaio clínico cruzado, no qual utilizou-se as técnicas de espirometria de incentivo a volume e breath stacking, verificaram que durante a realização desta última técnica houve maior mobilização de volume inspirado, e no pós-operatório, observou-se uma redução significativa dos volumes inspiratórios em ambas as técnicas, sendo mais acentuada na espirometria de incentivo. Já para Chinali et al. ${ }^{27}$, ao realizarem o ensaio clínico não controlado, em que seus pacientes foram divididos em um grupo para terapia com padróes ventilatórios (diafragmático, inspiração em tempos e máxima sustentada) e em outro grupo para inspirômetro de incentivo orientado a fluxo, notaram que não houve diferenças significativas nos valores de capacidade vital forçada (CVF), volume expiratório forçado no primeiro segundo $\left(\mathrm{VEF}_{1}\right)$ e pressão inspiratória máxima (PImáx) entre os grupos, embora as técnicas contribuam para suas melhoras. Indivíduos ventilados mecanicamente durante as CAA apresentam variações na função pulmonar no PO, como queda da CVF e VEF.

Treschan et al. 18 desenvolveram pesquisa para comparar a função pulmonar dos pacientes submetidos à CAA, com VM (ventilação mecânica) em altos e baixos VC. Consistiu-se um ensaio clínico duplo-cego, prospectivo, randomizado e controlado, com amostra de 101 pacientes, divididos em dois grupos, um que recebeu VC de $6 \mathrm{ml} / \mathrm{kg}$ de peso predito (PP) e outro que foi ventilado com VC de $12 \mathrm{ml} / \mathrm{kg}$ PP, havendo PEEP 
(positive end-expiratory pressure ou pressáo positiva expiratória final) de $5 \mathrm{cmH} 2 \mathrm{O}$ em ambos os grupos. A pesquisa provou que não houve diferenças significativas nos valores de CVF ou VEF1 entre os grupos nos primeiros cinco dias de $\mathrm{PO}$. Assim, VC baixos não amenizam os efeitos negativos do sistema respiratório decorrente da CAA.

Zhang et al. ${ }^{32}$, em estudo randomizado, fizeram divisáo de 203 pacientes em grupo experimental (submetidos à terapia com $\mathrm{PEP}$ - pressão expiratória positiva -oscilatória) e grupo controle (submetidos a cuidados usuais). Apresentou-se redução dos casos de febre e tempo de internação dos sujeitos do primeiro grupo. Os autores reforçam que os efeitos do método são incertos, necessitando assim de novas pesquisas.

Em contrapartida, Possa et al. ${ }^{30}$ desenvolveram uma diretriz de tratamento em fisioterapia para paciente no PO de CAA, através de um desenho de estudo de "antes e depois" com controle histórico. Para a elaboração do programa foi priorizado estratégias adicionais, como EPAP ou CPAP (continuous positive dirway pressure ou pressão positiva contínua na via aérea), espirometria de incentivo e mobilização precoce. Durante a aplicação, foi usado o EPAP em todos os pacientes que aderiram ao protocolo. Assim, apesar de sua utilização ser ainda bastante discutida, a redução da taxa de atelectasia encontrada na pesquisa pode ser justificada como resultado da utilização desse recurso.

Em um estudo turco, investigaram-se os efeitos do uso do CPAP e da pressão positiva nas vias aéreas em dois níveis (BiPAP - bilevel positive airway pressure) na mecânica respiratória. A administração profilática do CPAP apresentou melhores efeitos na $\mathrm{PaO}_{2}$ e $\mathrm{SpO}_{2}$, numa comparação ao BiPAP e oxigenoterapia ${ }^{33}$.

No estudo realizado por Forgiarini Junior et al. ${ }^{4}$, em que 36 pacientes foram submetidos às cirurgias abdominais, observou-se que aqueles indivíduos que passaram por um atendimento precoce da fisioterapia respiratória, ou seja, já na sala de recuperação pós-anestésica, obtiveram menores variaçóes em relação aos valores de força muscular respiratória no pós-operatório, com PEmáx (pressão expiratória máxima): $53,4 \mathrm{cmH}_{2} \mathrm{O} \pm$ 10,9 , numa comparação aos que foram atendidos somente na enfermaria, com PEmáx de $40,1 \mathrm{cmH}_{2} \mathrm{O} \pm$ 7,6. Entretanto, não foi possível identificar que tipo de tratamento fisioterapêutico o autor utilizou para desenvolver seu trabalho. Para Silva et al. ${ }^{10}$, em pesquisa com pacientes que realizaram cirurgia de laparatomia e tiveram acompanhamento fisioterapêutico, houve um aumento significativo da PEmáx, uma vez que no pré-tratamento os pacientes obtiveram PEmáx de 43,15 $\mathrm{cmH}_{2} \mathrm{O} \pm 22$, e no pós-tratamento, PEmáx de 49,10 $\mathrm{cmH}_{2} \mathrm{O} \pm 22$.
Hanekom et al. ${ }^{29}$ destacam a inclusão na terapêutica de técnicas para remoção de secreção, como a tosse. Além das outras técnicas já citadas, seu estudo também ressalta a importância da mobilização precoce, da associação de técnica de expiração forçada e CPAP e da aspiração em última escolha para o tratamento de secreção nesses pacientes. Destaca ainda a importância da experiência clínica do terapeuta para a tomada de decisão, que deve ser baseada em evidências, ainda que escassas. Assim, a fisioterapia pode integrar o tratamento no PO através de técnicas e métodos para reduzir ou prevenir complicaçóes funcionais em pacientes de cirurgia abdominal alta $^{34}$.

A partir deste estudo foi possível identificar que a abordagem fisioterapêutica, embora aceita no tratamento do PO de pacientes que realizaram uma CAA, ainda carece de pesquisas que comprovem sua eficácia com resultados mais conclusivos, que possam servir para a tomada de decisão sobre qual terapia deve ser utilizada para cada caso. Considerando as principais limitaçóes, os estudos mostraram-se inconclusivos e com amostras pequenas, abrindo assim vieses de comprometimento dos resultados. Ressalta-se ainda, a necessidade de estudos randomizados mais conclusivos com amostras maiores e métodos bem definidos.

\section{Conclusão}

A partir dos estudos revisados foi possível discutir as principais técnicas de fisioterapia respiratória utilizadas no $\mathrm{PO}$ de CAA, entretanto não foi possível eleger uma terapia que seja essencial para o tratamento. Todavia, todos os estudos ressaltam a importância da fisioterapia para redução ou prevenção de complicações pulmonares, e que ela deve ser iniciada precocemente para que se reduza o tempo de permanência hospitalar com consequente redução dos gastos médicos.

\section{Referências}

1. Martins FS. Complicações pulmonares pós-operatórias de cirurgias abdominais altas e abordagem fisioterapêutica [monografia]. -Tubarão (SC): Universidade do Sul de Santa Catarina; 2005.

2. Boden I, Browning L, Skinner EH, Reeve J, El-Ansary D, Robertson IK, et al. The LIPPSMAck POP (Lung Infection Prevention Post Surgery - Major Abdominal - with PreOperative Physiotherapy) trial: study protocol for a multi-centre randomised controlled trial. Trials. 2015;16(1):573.

3. Arruda KA, Cantaneo DC, Cataneo AJM. Surgical risk tests related to cardiopulmonar postoperative complications. 
Comparison between upper abdominal and thoracic surgery. Acta Cir Bras. 2013;28(6):458-66.

4. Forgiarini Júnior LA, Carvalho AT, Ferreira TS, Monteiro MB, Dal Bosco A, Gonçalves MP, et al. Atendimento fisioterapêutico no pós-operatório imediato de pacientes submetidos à cirurgia abdominal. J Bras Pneumol. 2009;35(5):455-59.

5. Dias CM, Plácido TR, Ferreira MFB, Guimarães FS, Menezes SLS. Inspirometria de incentivo e breath stacking: repercussóes sobre a capacidade inspiratória em indivíduos submetidos à cirurgia abdominal. Rev Bras Fisioter. 2008;12(2):94-9.

6. Kumar AS, Alaparthi GK, Augustine AJ, Pazhyaottayil ZC, Ramakrishna A, Krishnakumar SK. Comparison of flow and volume incentive spirometry on pulmonary function and exercise tolerance in open abdominal surgery: a randomized clinical trial. J Clin Diagn Res. 2016;10(1):1-6.

7. Sarmento LF, Pinto V. Fisioterapia e as complicações pulmonares no pós-operatório de esofagectomia: uma revisão integrativa da literatura. Assobrafir Ciência, 2014;5(3):63-80.

8. Andreollo NA, Lopes LR, Coelho Neto JS. Complicaçóes pós-operatórias após gastrectomia total no câncer gástrico: análise de 300 doentes. Arq. Bras. Cir. Dig. 2011;24(2):126-30.

9. Caobianco JDR, Souza Filho CM, Bittencourt WS, Salicio MA, Salício VAMM. Estudo de revisão sobre o tempo de recuperação da função respiratória em pacientes submetidos à Cirurgia Abdominal Alta. Uniciências. 2010;14(2):287-301.

10. Silva FA, Lopes TM, Duarte J, Medeiros RF. Tratamento fisioterapêutico no pós-operatório de laparotomia. J Health Sci Inst. 2010;28(4):341-4.

11. Santos A. Influência do treino dos músculos inspiratórios na capacidade ventilatória no período pós-operatório de colecistectomia laparoscópica [monografia]. Porto (Portugal): Universidade Fernando Pessoa; 2011.

12. Santos CA, Rabelo BAS, Borges DL, Silva MGB, Silva TM. Avaliação da força muscular respiratória de pacientes submetidos à colecistectomia videolaparoscópica. Assobrafir Ciência. 2016;7(1):35-42.

13. Lunardi AC, Miranda CS, Silva KM, Cecconello I, Carvalho CRF. Weakness of expiratory muscles and pulmonary complications in malnourished patients undergoing upper abdominal surgery. Respirology. 2012;17(1):108-13.

14. Paisani DM, Chiavegato LD, Faresin SM. Volumes, capacidades pulmonares e força muscular respiratória no pós-operatório de gastroplastia. J Bras Pneumol. 2005;31(2):125-32.

15. Sakai RL, Abrão GMG, Ayres JFV, Vianna PTG, Carvalho LR, Castiglia YMM. Prognostic factors for perioperative pulmonar events among patients undergoing upper abdominal surgery. Sao Paulo Med J. 2007;125(6):315-21.

16. Filardo FA, Faresin SM, Fernandes ALG. Validade de um índice prognóstico para ocorrência de complicaçóes pulmonares no pós-operatório de cirurgia abdominal alta. Rev Assoc Med Bras. 2002; 48(3):209-16.

17. Tomich GM, França DC, Diniz MTC, Britto RR, Sampaio RF, Parreira VF. Efeitos de exercícios respiratórios sobre o padrão respiratório e movimento toracoabdominal após gastroplastia. J Bras Pneumol. 2010;36(2):197-204.

18. Treschan TA, Kaisers W, Schaefer MS, Bastin B, Schmalz U, Wania V, et al. Ventilation with low tidal volumes during upper abdominal surgery does not improve postoperative lung function. Br J Anaesth. 2012;109(2):263-71.

19. Grams ST, Ono LM, Noronha MA, Schivinski CIS, Paulin E. Breathing exercises in upper abdominal surgery: a systematic review and meta-analysis. Rev Bras Fisioter. 2012;16(5):345-53.

20. Cangussu DDD. Avaliação de volumes, capacidade e força muscular respiratória em pacientes submetidos à cirurgia abdominal alta eletiva [dissertação]. Brasília, DF: Universidade Católica de Brasília; 2006.

21. Ueda H, Hoshi T. Aumento da capacidade residual funcional durante cirurgia laparoscópica com elevação da parede abdominal. Rev Bras Anestesiol. 2017;67(3):284-7.

22. Manzano RM, Carvalho CRF, Saraiva-Romanholo BM, Vieira JE. Chest physiotherapy during immediate postoperative period among patients undergoing upper abdominal surgery: randomized clinical trial. São Paulo Med J. 2008; 126(5):269-73.

23. Fernandes SCS, Santos RS, Giovanetti EA, Taniguchi C, Silva CSM, Eid RAC, et al. Impacto da fisioterapia respiratória na capacidade vital e na funcionalidade de pacientes submetidos à cirurgia abdominal. Einstein (São Paulo). 2016;14(2):202-7.

24. Barbalho-Moulim MC, Miguel GPS, Forti EMP, Costa D. Comparação entre inspirometria de incentivo e pressão positiva expiratória na função pulmonar após cirurgia bariátrica. Fisioterapia e Pesquisa. 2009;16(2):166-72.

25. Chinali C, Busatto HG, Mortari DM, Rockenbach CWF, Leguisamo CP. Inspirometria de incentivo orientada a fluxo e padrôes ventilatórios em pacientes submetidos a cirurgia abdominal alta. Conscientiae Saúde. 2009;8(2):203-10.

26. Trevisan ME, Soares JC, Rondinel TZ. Efeitos de duas técnicas de incentivo respiratório na mobilidade toracoabdominal após cirurgia abdominal alta. Fisioterapia e Pesquisa. 2010;17(4):322-6.

27. Hanekom SD, Brooks D, Denehy L, Fagevik-Olsén M, Hardcastle TC, Manie S, et al. Reaching consensus on the physiotherapeutic management of patients following upper abdominal surgery: a pragmatic approach to interpret equivocal evidence. BMC Med Inform Decis Mak. 2012;12(1):5.

28. Gastaldi AC, Magalhães CMB, Baraúna MA, Silva EMC, Souza HCD. Benefícios da cinesioterapia respiratória no pós-operatório de colecistectomia laparoscópica. Rev Bras Fisioter. 2008;12(2):100-6. 
29. Ribeiro S, Gastaldi AC, Fernandes C. The effect of respiratory kinesiotherapy in patients undergoing upper abdominal surgery. Einstein. 2008;6(2):166-9.

30. Possa SS, Amador BA, Costa AM, Sakamoto ET, Kondo CS, Vasconcellos ALM, et al. Implementation of a guideline for physical therapy in the postoperative period of upper abdominal surgery reduces the incidence of atelectasis and length of hospital stay. Rev Port Pneumol. 2014;20(2):69-77.

31. Zancan M, Fozza FC. Fisioterapia no pré e pós-operatório de cirurgia abdominal e torácica. EFDeportes. 2013;18(179):1.

32. Zhang XY, Wang Q, Zhang S, Tan W, Wang Z, Li J. The use of a modified, oscillating positive expiratory pressure device reduced fever and length of hospital stay in patients after thoracic and upper abdominal surgery: a randomised trial. J Physiother. 2015;61(1):16-20.

33. Yağlığlu H, Köksal GM, Erbabacan E, Ekici B. Comparison and evaluation of the effects of administration of postoperative non-invasive mechanical ventilation methods (CPAP and BiPAP) on respiratory mechanics and gas exchange in patients undergoing abdominal surgery. Turk J Anaesthesiol Reanim. 2015;43(4):246-52.

34. Smith PR, Baig MA, Brito V, Bader F, Bergman MI, Alfonso A. Postoperative pulmonary complications after laparotomy. Respiration. 2010;80(4):269-74.

35. Joia Neto L, Thomson JC, Cardoso JR. Complicaçôes respiratórias no pós-operatório de cirurgias eletivas e de urgência e emergência em um Hospital Universitário. J Bras Pneumol. 2005;31(1):41-7.

\section{Como citar este artigo:}

Silva DCB, Silva Filho LS. Fisioterapia respiratória no pós-operatório de cirurgia abdominal alta: uma revisão de literatura. Rev. Aten. Saúde. 2018;16(55):115-123. 